\title{
52 CHARACTERIZATION OF THE TUMOR MICROENVIRONMENT IN MELANOMA USING MULTIPLEXED ION BEAM IMAGING (MIBI)
}

${ }^{1} J a s o n$ Ptacek*, ${ }^{2}$ Matthew Vesely, ${ }^{2}$ David Rimm, ${ }^{1}$ Monirath Hav, ${ }^{1}$ Murat Aksoy, ${ }^{1}$ Ailey Crow, ${ }^{1}$ Jessica Finn. ${ }^{1}$ Ionpath, Inc, Menlo Park, CA, USA; ${ }^{2}$ Yale University School of Medicine, New Haven, CT, USA

Background The complexity of the tumor microenvironment (TME) necessitates the application of high-dimensional methods that can spatially resolve the phenotypic heterogeneity that exists within tumors. MIBI, which combines time-of-flight secondary ion mass spectrometry (ToF-SIMS) with metal labeled antibodies to simultaneously image $40+$ proteins at subcellular spatial resolution, was used to classify cell populations and their expression of immunoregulatory proteins within 54 melanoma samples.

Methods A tissue microarray (TMA) comprised of $0.6 \mathrm{~mm}$ FFPE melanoma cores was stained with a panel of 30 metal labeled antibodies. The tissue was imaged using MIBI and multi-step processing was performed to create images of the samples. Single cell segmentation enabled enumeration of 32 cell populations and quantitative analyses were performed of both immune checkpoint expression and the spatial relationships between cells of different types.

Results Tumor cells and immune cells represented 62\% (1.4\% - 92.2\%) and $24 \%(2.0 \%$ - 92.1\%), respectively, of the segmented cells from the melanoma samples. Fibroblasts, lymphatics, and blood vessels were also present at varying densities. Within the immune compartment, M2 macrophages, M2 monocytes, and monocyte-derived dendritic cells (mDCs) were most abundant, representing 36.3\% (4.9\% - 79.3\%), $7.0 \%(0.0 \%-30.6 \%)$, and $9.8 \%(0.0 \%-34.6 \%)$, respectively, of the total immune cell infiltrate. Rare populations such as myeloid-derived suppressor cells (MDSCs) and regulatory $\mathrm{T}$ cells were at greater than 100 cells $/ \mathrm{mm}^{2}$ in six samples and four samples, respectively. The presence of immune checkpoint markers (IDO-1, LAG3, PD-1, PD-L1, TIM-3) varied between populations and between samples. A minority of the samples showed expression of IDO-1 and PD-L1 on myeloid populations. Interestingly, among the myeloid subsets, M2 macrophages and monocyte-derived dendritic cells showed the most abundant PD-L1 expression. Although many samples had few $\mathrm{T}$ cell infiltrates, those that did showed expression of PD-1, LAG-3, and TIM-3 on T cell populations.

Conclusions MIBI offers high-parameter tissue imaging, at sensitivity and resolution suited to understanding the complex tumor immune landscape, including the spatial relationships of immune and tumor cells and the expression of immunoregulatory proteins. Datasets that quantify population densities and immune checkpoint expression levels across tumor samples, such as the present one, can be used to test for associations to clinical variables and further understand the TME at the cellular level.

http://dx.doi.org/10.1136/jitc-2021-SITC2021.052 